\title{
A checklist and conservation status of vascular plants in the Limestone forest of Metropolitan Ilocos Norte Watershed Forest Reserve, Northwestern Luzon, Philippines
}

\author{
MAE ANN R. BATUYONG ${ }^{1,4, \boldsymbol{v}}$, MICHAEL A. CALARAMO ${ }^{2}$, GRECEBIO JONATHAN D. ALEJANDRO ${ }^{1,3}$ \\ ${ }^{1}$ The Graduate School, University of Santo Tomas, España Blvd., 1015 Manila, Philippines. ”email: www.mrbatuyong@mmsu.edu.ph \\ ${ }^{2}$ Northwestern University Ecological Park \& Botanic Gardens. Airport Avenue, Bengcag, Laoag City, 2900, Ilocos Norte, Philippines \\ ${ }^{3}$ College of Science and Research Centre for the Natural \& Applied Sciences, University of Santo Tomas, España Blvd., 1015 Manila, Philippines \\ ${ }^{4}$ Department of Biological Sciences, College of Arts and Sciences, Mariano Marcos State University, City of Batac, 2906, Ilocos Norte, Philippines
}

Manuscript received: 8 June 2020. Revision accepted: 6 August 2020.

\begin{abstract}
Batuyong MAR, Calaramo MA, Alejandro GJD. 2020. A checklist and conservation status of vascular plants in the Limestone forest of Metropolitan Ilocos Norte Watershed Forest Reserve, Northwestern Luzon, Philippines. Biodiversitas 21: 3969-3981. The Metropolitan Ilocos Norte Watershed Forest Reserve (MINWFR) is among the remaining intact limestone formations and a critical protected area in northwestern Luzon. There have been few published floristic studies despite its undeniable rich biological importance. Therefore, this paper primarily aims to provide a preliminary checklist of vascular plants in MINWFR and their conservation status. Consequent field visits and surveys were made from April 2019 to March 2020. Results revealed a total of 173 species distributed in 140 genera belonging to 59 families. The most represented families are Rubiaceae, Fabaceae, Orchidaceae, and Malvaceae. This forest supports $49 \%$ or $28.65 \%$ local endemics, five of which are narrow endemics, namely Cyanometra warburgii, Pyrostria triflora, Syzygium ilocanum, Thrixspermum nicolasiorum, and Antirhea microphylla. The latter, however, extends up to Ilocos Sur. Based on IUCN criteria and DENR (Department of Environment and Natural Resources of Philippines) records, a total of 18 species are threatened, one species is recorded as critically endangered, five endangered, nine vulnerable, three other threatened, two near threatened, 55 as least concern, and the rest are not evaluated. Other noteworthy species present in the area are the two dominant endangered species, Podocarpus costalis, and Podocarpus polystachyus. An interesting spinescent Rubiaceae species were recorded and currently under examination to establish its identity. This checklist serves as a basis to effectively manage this vulnerable area surrounded by human-induced disturbances and threats.
\end{abstract}

Keywords: Biodiversity, conservation, Ilocos Norte, Limestone forest

\section{INTRODUCTION}

The Philippines supports many endemic and various species from different ecosystems owing to its highly diverse topography, climate patterns, and complex geological history (Myers et al. 2000; Yap 2010; Brown et al. 2013; Dapar et al. 2020). It is listed as one of the megadiverse countries (DENR-BMB 2015; CI 2020). However, it is reported as one of the top countries affected by biodiversity loss, thus making it one of the global conservation priority areas (Rockstrom et al. 2009; Mittermeier et al. 2011; CBD 2020).

In the past years, there have been increased government initiatives recompensed by conservation successes such as the establishments and declarations of Key Biodiversity Areas (KBAs) and Protected Areas (PAs) as cornerstones to ensure biodiversity conservation in the face of threats and pandemic we are experiencing (Sinsin 2012; DENRBMB 2015). Sadly, due to incessant deforestation and exploitations of several crucial habitats, this has led the country to be on the edge of ecological degradation and biodiversity collapse (Posa et al. 2008; Amoroso 2011; CBD 2020). One of the diverse types of forest ecosystems in the country that is gaining the need for top priority biodiversity research and urgent consideration for protection is limestone ecosystem. It is identified as a conservation focus for sustainability, especially that natural and anthropogenic disturbances are increasing (Sodhi et al. 2010). The Philippines is endowed with about $11.7 \%$ or extensive 35,000 $\mathrm{km}^{2}$ karst total land (DENR-BMB 2019), but a large portion of this is still unknown to science. The Philippines' Biodiversity Management Bureau recognized the need for a strategic action plan to save and sustainably managed this unique ecosystem as it hosts diverse flora and fauna. The growing interest in this type of forest formation is because of its panoramic view, ecotourism and recreational value, the potential discovery of new flora and fauna, the utilization of its mineral and aquifer resources, and cultural importance.

Among the considered remaining intact and important limestone areas found in Luzon (Tolentino et al. 2020), is the Metropolitan Ilocos Norte Watershed Forest Reserve (MINWFR). It is part of the Ilocos Mountains and described as one of the critical ecosystems by Calaramo et al. (2016). It has numerous cavern systems, and most of them contain subterranean rivers (DENR-MINWFR Management Plan 2018). Initially, it is established as a component by the National Integrated Protected Area 
System (NIPAS) through Presidential Proclamation No. 731, dated September 27, 1934, but currently, proposed to be a regular component.

Unfortunately, like any other area, its ecological aspects are also the most vulnerable and fragile (Brinkmann \& Parise 2012) and under immense pressure because of the observed disturbances in all corners of the PA brought by human exploitation and numerous infrastructure developments, aggravated by the harsh climatic condition. Meager information is also available about the richness and status of plants existing in this area. Hence, this suggests an imminent need to perform a floristic survey to increase the plant diversity knowledge on this limestone forest. As underscored by Ong et al. (2002), the conduct of floristic inventories through and mapping of habitat types must be undertaken, since these areas contain specific vegetation assemblages and may be very useful in predicting and validating plant distribution. Moreover, Stockli et al. (2011) emphasized that they are crucial elements in the monitoring of programs directed at looking into the effects of climate change.

Further, they will serve as essential guides necessary for creating conservation plans and programs directed for policy formulation (Bacudo et al. 2006; UNEP 2012; PCARRD 2017) and the practical management of the area in a way where potentially invasive species may be efficiently identified, accordingly monitored, and properly contained. Thus, this study provides a preliminary checklist of vascular plants, including their conservation status in the limestone forest of MIWFR, Northwestern Luzon, Philippines.

\section{MATERIALS AND METHODS}

\section{Study site}

The study site is positioned geographically in the Metropolitan Ilocos Norte Watershed Forest Reserve (MINWFR), Municipality of Pasuquin, Province of Ilocos Norte, Philippines (Figure 1). Based on the DENR (Department of Environment and Natural Resources of Philippines) cadastral survey, it has an estimated land area of 2,075 hectares with a buffer zone of 412 hectares. It lies between geographical coordinates of $120^{\circ} 37^{\prime} 00^{\prime \prime}-120^{\circ}$ $41^{\prime} 00^{\prime \prime}$ North and $18^{\circ} 21^{\prime} 00^{\prime \prime}-18^{\circ} 24^{\prime} 00^{\prime \prime}$ East. It covers Barangays Sapat, Tadao, Salpad, Sulbec, Sulongan, Susugaen, and Estancia.

The climate of MINWFR sits in the type 1 climate with pronounced dry and wet season based on the Corona Classification. There is a severe dryness during the summer months from January to May, which reaches a very high temperature $\left(39^{\circ} \mathrm{C}\right)$. The wet months from June to October transform the vegetation into greenery, where trees create a thick canopy, and numerous seasonal herbs grow on its rocky ecosystems. Tropical cyclones and storms occasionally visit the Municipality of Pasuquin during the southwest monsoon season (May to October), and it is most dominant during July. Exposed to Southeast monsoon and cyclonic rains, this area experiences prevailing wind blowing from north to south and sometimes west to east, primarily when rainfall occurs. However, the area is shielded from the northeast and trade winds by the Northern Cordillera Ranges.

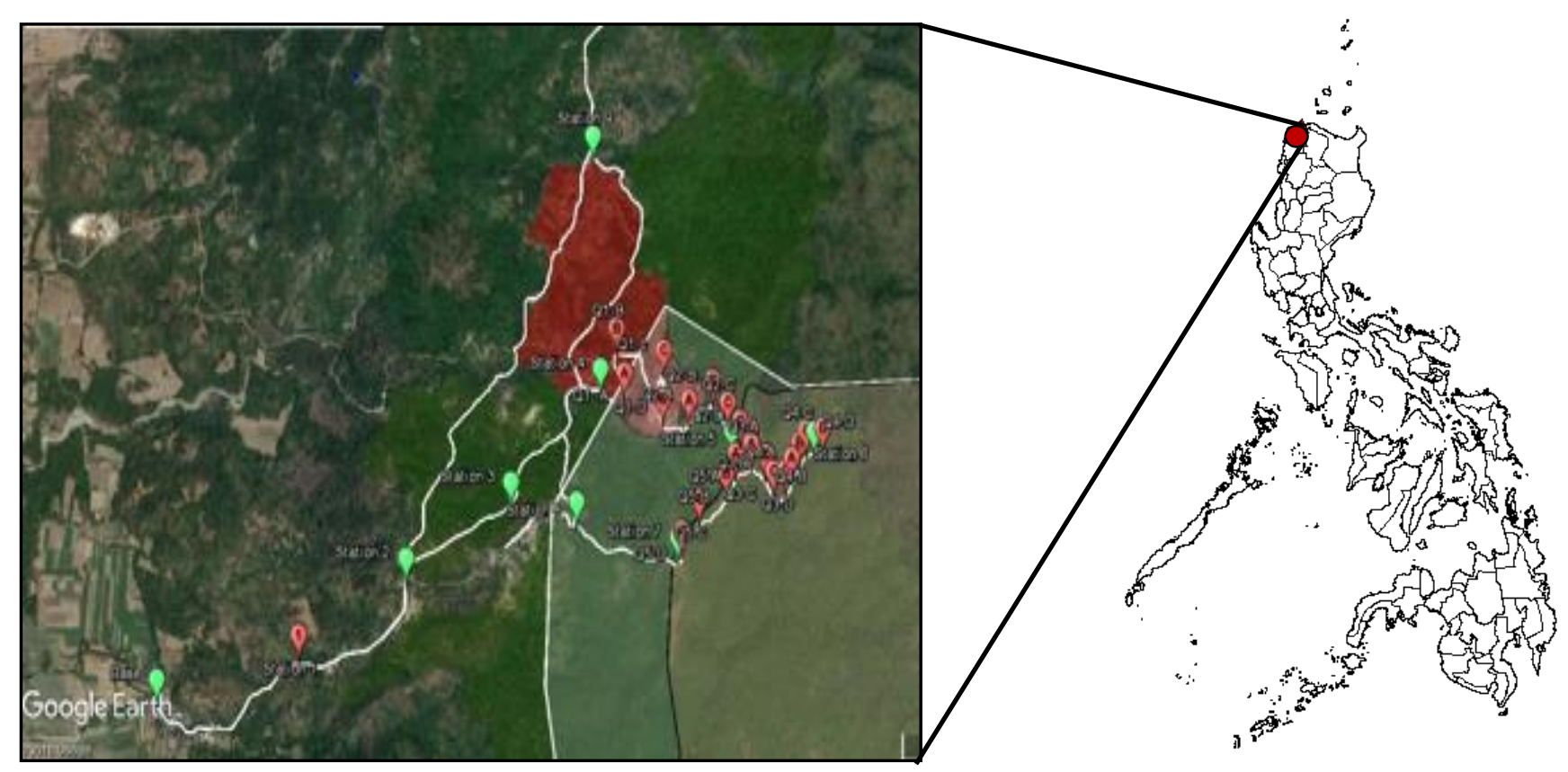

Figure 1. Map of the study sites in Metropolitan Ilocos Norte Watershed Forest Reserve (MINWFR), Northwestern Luzon, Municipality of Pasuquin, Province of Ilocos Norte, Philippines. Surveyed areas are marked in green and red 
MINWFR is generally mountainous with rugged terrain. Terrain ranges from $0-8 \%$ level to undulating, 8 $18 \%$ undulating to rolling, $18-30 \%$ rolling to hilly, $30-50 \%$ hilly to mountainous, and $50 \%$ very steep. The highest elevation of the protected area is 539 meters above sea level (asl). The highest altitude in the Municipality is 564 meters located within the military reserve, which is directly adjacent to the PA. MINWFR mainly consists of Bolinao Clay with relatively small portions of San Fernando Clay Loam and Umingan Clay Loam (DENR-MINWFR Management Plan 2018).

\section{The survey, collection, and processing of specimens}

Before the actual field survey and to fulfill the legal requirements of EO 247 (Bioprospecting) and RA 9174 (Wildlife Resource Conservation and Protection Act), a research proposal was presented to the members of the Protected Area Management Board (PAMB) of MINWFR for their approval and eventual issuance of the Gratuitous Permit from the Department of Environment and Natural Resources of Region 1.

The consequent field visit was made in the area from April to October of 2019 to map and identify the sites and trails, and for the initial listing and photo documentation of plant species in the area. Then, the actual surveys, data gathering, and the collection were conducted in November 2019 to February 2020, where there are more observed plants that are in their flowering and/or fruiting stage. A revisit in the area was done in March 2020 for the final documentation of plants with their mature fruits.

The plant specimens were collected and assessed along and approximately $10 \mathrm{~m}$ in the accessible and identified trails in the site (Figure 1) as surveyed areas. Representative specimens collected were pressed, cured, and mounted following standard procedures for herbarium vouchers. Field data as to the habitat, physiognomy, characteristics of the plants that will be lost after drying, local names, and coordinates were noted. Herbarium specimens were labeled and kept at the Herbarium of Northwestern Luzon (HNUL) and University of Santo Tomas Herbarium (USTH).

\section{Identification of plant materials}

Relevant literature such as Enumeration of Philippine Flowering Plants (Merrill 1925) and some open-access websites such as Co's Digital Flora of the Philippines (www.philippineplants.org), or digital herbarium specimen was accessed mainly through JSTOR (https://plants.jstor.org/) and the Global Biodiversity Information Facility, GBIF (http://www.gbif.org/) to confirm for the identification. The collections from the following local herbaria such as National Museum of the Philippines (PNH), University of the Philippines Los Banos- Museum of Natural History (CAHUP), University of Santo Tomas-Herbarium (USTH), and Herbarium of Northwestern Luzon (HNUL) were also accessed for the validation of the identity of the collected plants.

The nomenclatural status of the species identified was reviewed through The Plant List (http://www.theplantlist.org) and International Plant Name
Index, IPNI (https://www.ipni.org/). Online databases such as The Global Biodiversity Information Facility ((http://www.gbif.org/)) and Co's Digital Flora (Pelser et al. 2011 onwards) of the Philippines were used to identify the occurrence and distribution of the plants collected. The classification of the conservation status was based on the International Union of Conservation of Nature (IUCN) Red List of Threatened Species (https://www.iucnredlist.org/) and the Updated National List of Threatened Plants in the Philippines (DENR DAO 2017-11) to determine the status, whether Critically Endangered (CR), Endangered (EN), Vulnerable (VU), Other Threatened (OT), Near Threatened (NT), Least Concern (LC), Data Deficient (DD) and Not Evaluated (NE).

\section{RESULTS AND DISCUSSION}

\section{Description of the vegetation and flora in the area}

The high floral richness recorded on forest over limestone is considered still as underestimated (Clements et al. 2006). Moreover, according to Tolentino et al. (2020), a few existing studies focusing on limestone forests in the country were conducted. The forest structure in MINWFR showed differences in vegetation just a few kilometers apart from one area to another; (i) some portions of the forest are open brushland, while others have been converted into agroforestry and reforestation areas, (ii) other areas are observed as seasonal dry with tall trees and small understory trees, (iii) to forested thickets with the presence of ferns, herbaceous plants, and shrubs in dense population with high growing trees, which makes the upper canopy, at the same time, limestone is present on the ground scape, (iv) while another site is dominated by sparse and small stunted trees over rocky ridges with numerous herbaceous plants on the ground, which indicate drought-tolerant population, some of the trees and shrubs tend to have leathery and thick leaves. This diverse ecological niches, specific vegetation and complex terrains that could be found existing within the limestone landscape (Clements et al. 2006; Saw et al. 2010; Tolentino et al. 2020 ) is similarly observed in the area. According to PerezGarcia et al. (2009), the observed high environmental heterogeneity found within the forest is attributed to its unusual soil condition. Taking also into consideration the geological history, hydrology, micro-climatic conditions, and the prevailing climate of the area as this forest vary from other types of rainforest, with regards to its structure, species richness as well as species composition (Whitmore 1984; Fernando et al. 2008).

The result of the plant survey done revealed that MINWFR has notable plant composition. Overall, the preliminary list of recorded families of vascular plants, ferns and their allies (angiosperms and gymnosperms) from the area composed a total of 173 species, 140 genera belonging to 59 families. The recorded species comprised of 106 small to large trees, 30 shrubs, 17 herbs, six vines, and 14 epiphytes (Table 1). 
Table 1. Taxonomic inventory of vascular plants documented in MINWFR, Northwestern Luzon, Philippines

\begin{tabular}{lccc}
\hline & \multicolumn{3}{c}{ Total number of } \\
\cline { 2 - 4 } & Families & Genera & Species \\
\hline Trees & 39 & 81 & 106 \\
Shrubs & 16 & 22 & 30 \\
Herbs & 11 & 16 & 17 \\
Vines & 2 & 3 & 6 \\
Epiphytes & 5 & 15 & 14 \\
\hline Total & & & 173 \\
\hline
\end{tabular}

Of the 59 families, two were gymnosperms represented by Cycadacea and Podocarpacea. The Angiosperm family having the most number of genera, is Rubiaceae (17), followed by Fabaceae (12), Orchidaceae (7), Lamiaceae, Malvaceae, Poaceae (6), and Phyllantaceae (4), while the rest of the families each having three or fewer genera (Table 2; Figure 4). Some of these families are among the included typical families observed in a forest over limestone (Pedregosa et al. 2006; Kiew 2014; Replan and Malaki 2017). The Rubiaceae represents the world's fourthlargest flowering plant family, after Orchidaceae, Asteraceae, and Fabaceae (Govaerts et al. 2006) that are widely distributed in the tropics. Besides, species of Rubiaceae were also found in the forest over limestone of
Eastern Samar Visayas based on a checklist by Ordas et al. (2018).

The dominant genera Ficus L. and Syzygium R. Br. ex Gaertn belonging to the family Moraceae and Myrtaceae, respectively, are highly represented, having four species each. Other genera with abundant species were Albizia Durazz. and Callophyllum L. having three species each. Whereas, a single genus represented 27 of the families or $45.76 \%$. This number of plants represents $1.70 \%$ out of the 10,158 species of vascular plants in the Philippines (Pelser et al. 2011 onwards).

Some of the frequently occurring species encountered were Antirhea microphylla (Bartl. ex DC.) Merr, Ixora philippinensis Merr., Psychotria luzoniensis (Cham. \& Schltdl.) Fern.-Vill, Syzygium simile (Merr.) Merr., Ziziphus talanai (Blanco) Merr. and the spinescent species that were not identified to the species level but assigned under the genus Canthium Lam. of Rubiaceae (Figure 2). This interesting species is currently being ascertained through morphological and molecular studies to establish its identity. The pitcher plant (Nepenthes alata Blanco) was also among the flora thriving in the area but restricted in one site.

Notably, the forest as well carries primitive species with the presence of Cycads and sprawling and abundant century-old trees Podocarpus costalis C. Presl and Podocarpus polystachyus R.Br. ex Endl.) (Figure 4).

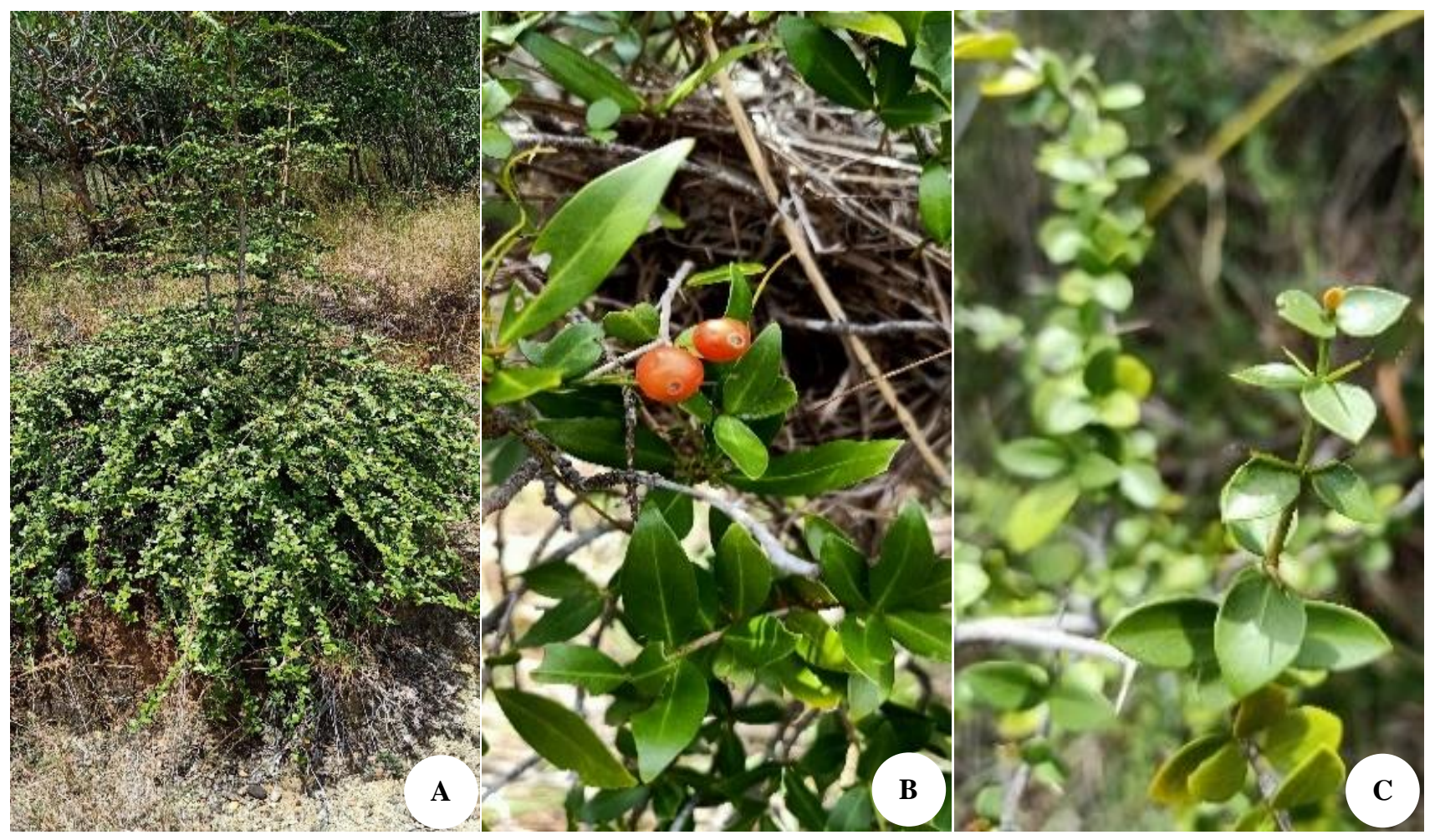

Figure 2. The unidentified Canthium sp. (Rubiaceae) collected in MINWFR, Northwestern Luzon, Philippines during the dry season (November). A. Whole plant, B. Flower bud and fruit at maturity, C. Close-up of the leaves showing the spines. 
Table 2. List of recorded species in MINFWR, Northwestern Luzon, Philippines

\begin{tabular}{|c|c|c|c|c|c|}
\hline Family & Species & Local name & Habit & $\begin{array}{c}\text { Conservation } \\
\text { Status } \\
\end{array}$ & Endemicity \\
\hline Acanthaceae & Strobilanthes viridis (Merr.) Y.F. Deng & & $\mathrm{H}$ & $\mathrm{NE}$ & $\mathrm{E}$ \\
\hline \multirow[t]{5}{*}{ Anacardiaceae } & Buchanania microphylla Engl. & & $\mathrm{T}$ & $\mathrm{NE}$ & $\mathrm{N}$ \\
\hline & Buchanania aborescens (Blume) Blume & & $\mathrm{T}$ & $\mathrm{NE}$ & $\mathrm{N}$ \\
\hline & Dracontomelon dao (Blanco) Merr. \& Rolfe & & $\mathrm{T}$ & VU* & $\mathrm{N}$ \\
\hline & Semecarpus cuneiformis Blanco & Ligas & $\mathrm{T}$ & $\mathrm{NE}$ & $\mathrm{N}$ \\
\hline & Semecarpus longifolius Blume & Anagas & $\mathrm{T}$ & $\mathrm{LC}$ & $\mathrm{N}$ \\
\hline \multirow[t]{3}{*}{ Annonaceae } & Goniothalamus amuyon (Blanco) Merr. & Amoyun & $\mathrm{T}$ & $\mathrm{NE}$ & $\mathrm{N}$ \\
\hline & $\begin{array}{l}\text { Pseudouvaria luzoniensis (Merr.) Y.C.F.Su \& } \\
\text { R.M.K.Saunders }\end{array}$ & Allagat & $\mathrm{T}$ & NE & $\mathrm{E}$ \\
\hline & Uvaria grandiflora Roxb. Ex Hornem & & $\mathrm{T}$ & $\mathrm{NE}$ & $\mathrm{N}$ \\
\hline \multirow[t]{6}{*}{ Apocynaceae } & Alstonia macrophylla Wall. Ex G.Don & Dalipawen & $\mathrm{T}$ & $\mathrm{LC}$ & $\mathrm{N}$ \\
\hline & Alyxia luzoniensis Merr. & & $\mathrm{V}$ & $\mathrm{NE}$ & $\mathrm{E}$ \\
\hline & Alyxia monticola C.B.Rob. & & $\mathrm{V}$ & $\mathrm{NE}$ & $\mathrm{N}$ \\
\hline & Dischidia luzonica (Schltr.) Arshed, Agoo \& Rodda & & $\mathrm{V}$ & $\mathrm{NE}$ & $\mathrm{E}$ \\
\hline & Hoya benguetensis Schltr. & & $\mathrm{V}$ & NE & $\mathrm{E}$ \\
\hline & Vincetoxicum elmeri (Schltr.) Meve \& Liede & & $\mathrm{V}$ & $\mathrm{NE}$ & $\mathrm{E}$ \\
\hline Araceae & Colocasia esculenta (L.) Schott. & Aba & $\mathrm{H}$ & $\mathrm{LC}$ & $\mathrm{N}$ \\
\hline Araliaceae & Polyscias nodosa (Blume) Seem. & & $\mathrm{T}$ & $\mathrm{LC}$ & $\mathrm{N}$ \\
\hline \multirow{4}{*}{ Arecaceae } & Areca catechu $\mathrm{L}$. & Bua & $\mathrm{T}$ & $\mathrm{NE}$ & $\mathrm{N}$ \\
\hline & Areca ipot Becc. & Bua & $\mathrm{T}$ & $\mathrm{VU} * / \mathrm{VU}$ & $\mathrm{E}$ \\
\hline & Caryota cumingii Lodd. ex C. Mart & Anibong & $\mathrm{T}$ & NE & $\mathrm{E}$ \\
\hline & Heterospathe cagayanensis Becc. & Bagobo & $\mathrm{T}$ & $\mathrm{NE}$ & $\mathrm{E}$ \\
\hline Asparagaceae & Dracaena multiflora Warb. ex Sarasin & & $\mathrm{T}$ & $\mathrm{LC}$ & $\mathrm{N}$ \\
\hline Begoniaceae & Begonia rufipila Merr. & & $\mathrm{H}$ & $\mathrm{NE}$ & $\mathrm{E}$ \\
\hline Bignoniaceae & Radermachera coriacea Merr. & & $\mathrm{T}$ & $\mathrm{VU}^{*}$ & $\mathrm{E}$ \\
\hline Burseraceae & Canarium asperum Benth. & Anteng & $\mathrm{T}$ & $\mathrm{LC}$ & $\mathrm{N}$ \\
\hline \multirow{3}{*}{ Calophyllaceae } & Calophyllum blancoi Planch. \& Triana & Pamitaogen & $\mathrm{T}$ & NE & $\mathrm{N}$ \\
\hline & Calophyllum inophyllum $\mathrm{L}$. & Bittaog & $\mathrm{T}$ & $\mathrm{LC}$ & $\mathrm{N}$ \\
\hline & Calophyllum pentapetalum (Blanco) Merr. & Bittaog bantay & $\mathrm{T}$ & NE & $\mathrm{E}$ \\
\hline \multirow[t]{2}{*}{ Cannabaceae } & Celtis philippinensis Blanco & & $\mathrm{T}$ & NE & $\mathrm{N}$ \\
\hline & Trema orientalis (L.) Blume & Agandong & $\mathrm{T}$ & $\mathrm{LC}$ & $\mathrm{N}$ \\
\hline \multirow[t]{2}{*}{ Capparaceae } & Capparis micracantha DC. & Pacpacaw-it & $\mathrm{S}$ & $\mathrm{NE}$ & $\mathrm{N}$ \\
\hline & Capparis sepiaria L. & & $\mathrm{S}$ & $\mathrm{NE}$ & $\mathrm{N}$ \\
\hline Casuarinaceae & Cassuarina equisetifolia $\mathrm{L}$. & Aro-o & $\mathrm{T}$ & $\mathrm{NE}$ & $\mathrm{N}$ \\
\hline Celastraceae & Salacia korthalsiana Miq. & & $\mathrm{S}$ & $\mathrm{NE}$ & $\mathrm{N}$ \\
\hline Clusiaceae & Garcinia binucao (Blanco) Choisy & & $\mathrm{T}$ & $\mathrm{NE}$ & $\mathrm{N}$ \\
\hline \multirow[t]{2}{*}{ Combretaceae } & Terminalia catappa $\mathrm{L}$. & Binucaw & $\mathrm{T}$ & LC & $\mathrm{N}$ \\
\hline & Terminalia nitens $\mathrm{C}$. Presl & Salaysay & $\mathrm{T}$ & VU & $\mathrm{N}$ \\
\hline Cycadaceae & Cycas riuminiana Porte. ex Regel & Parangipang & $\mathrm{T}$ & $\mathrm{VU} * / \mathrm{E}$ & $\mathrm{E}$ \\
\hline \multirow[t]{2}{*}{ Dipterocarpaceae } & Shorea astylosa Foxw. & Yakal & $\mathrm{T}$ & $\mathrm{CR} * / \mathrm{E}$ & $\mathrm{E}$ \\
\hline & Shorea contorta S.Vidal & White Lauan & $\mathrm{T}$ & LC & $\mathrm{E}$ \\
\hline Droseraceae & Drosera burmannii Vahl & & $\mathrm{H}$ & $\mathrm{NE}$ & $\mathrm{N}$ \\
\hline \multirow[t]{3}{*}{ Ebenaceae } & Diospyrus maritima Blume & & $\mathrm{T}$ & $\mathrm{NE}$ & $\mathrm{N}$ \\
\hline & Diospyrus montana var. timorensis Bakh. & Ballatinaw & $\mathrm{T}$ & $\mathrm{NE}$ & $\mathrm{E}$ \\
\hline & Diospyros pilosanthera Blanco & Bolong-eta & $\mathrm{T}$ & VU* & $\mathrm{N}$ \\
\hline Erythroxylaceae & Erythroxylum cuneatum (Miq.) Kurz & & $\mathrm{T}$ & $\mathrm{NE}$ & $\mathrm{N}$ \\
\hline \multirow{3}{*}{ Euphorbiaceae } & Acalypha cardiophylla Merr. & & $\mathrm{S}$ & $\mathrm{LC}$ & $\mathrm{N}$ \\
\hline & Macaranga tanarius (L.) Müll. Arg. & Samac & $\mathrm{T}$ & $\mathrm{LC}$ & $\mathrm{N}$ \\
\hline & Mallotus philippensis (Lam.) Müll & & $\mathrm{T}$ & $\mathrm{LC}$ & $\mathrm{N}$ \\
\hline \multirow[t]{15}{*}{ Fabaceae } & Abrus precatorius L. & Bugayong & $\mathrm{H}$ & $\mathrm{NE}$ & $\mathrm{N}$ \\
\hline & Acacia auriculiformis A.Cunn. ex Benth. & Auri & $\mathrm{T}$ & $\mathrm{LC}$ & $\mathrm{N}$ \\
\hline & Acacia mangium Willd. & Mangium & $\mathrm{T}$ & $\mathrm{LC}$ & $\mathrm{N}$ \\
\hline & Albizia lebbekoides (DC.) Benth. & Carisquis & $\mathrm{T}$ & $\mathrm{LC}$ & $\mathrm{N}$ \\
\hline & Albizia procera (Roxb.) Benth. & Adaan & $\mathrm{T}$ & $\mathrm{LC}$ & $\mathrm{N}$ \\
\hline & Albizia saman (Jacq.) F. Muell. & Algarlubo & $\mathrm{T}$ & $\mathrm{NE}$ & $\mathrm{N}$ \\
\hline & Cynometra warburgii Harms & Marabalitbitan & $\mathrm{T}$ & $\mathrm{NE}$ & $\mathrm{E}$ \\
\hline & Entada phaseoloides (L.) Merr. & Lipay & $\mathrm{T}$ & OTS* & $\mathrm{N}$ \\
\hline & Gliricidia sepium (Jacq.) Kunth ex Steud. & Cacaoate & $\mathrm{T}$ & LC & $\mathrm{N}$ \\
\hline & Leucaena leucocephala (Lam.) de Wit & Ipil-ipil & $\mathrm{T}$ & $\mathrm{NE}$ & $\mathrm{N}$ \\
\hline & Mimosa pudica L. & Bain-bain & $\mathrm{H}$ & LC & $\mathrm{N}$ \\
\hline & Pterocarpus indicus Willd & Dungon & $\mathrm{T}$ & EN & $\mathrm{N}$ \\
\hline & Senna spectabilis (DC.) H.S.Irwin \& Barneby & & $\mathrm{T}$ & $\mathrm{LC}$ & $\mathrm{N}$ \\
\hline & Tamarindus indica $\mathrm{L}$. & Salamagi & $\mathrm{T}$ & $\mathrm{LC}$ & $\mathrm{N}$ \\
\hline & Vachellia farnesiana (L.) Wight \& Arn. & Kandaruma & $\mathrm{T}$ & $\mathrm{LC}$ & $\mathrm{N}$ \\
\hline
\end{tabular}




\begin{tabular}{|c|c|c|c|c|c|}
\hline \multirow{7}{*}{ Lamiaceae } & Clerodendrum minahassae Teijsm. \& Binn. & Bagawac puraw & $\mathrm{S}$ & $\mathrm{NE}$ & $\mathrm{N}$ \\
\hline & Gmelina elliptica $\mathrm{Sm}$. in Rees & Melina & $\mathrm{T}$ & $\mathrm{LC}$ & $\mathrm{N}$ \\
\hline & Mesosphaerum suaveolens (L.) Kuntze & Mara-erva & $\mathrm{H}$ & NE & $\mathrm{N}$ \\
\hline & Premna odorata Blanco & Alagao & $\mathrm{T}$ & NE & $\mathrm{N}$ \\
\hline & Tectona grandis L.f & Teak & $\mathrm{T}$ & NE & $\mathrm{N}$ \\
\hline & Vitex negundo $\mathrm{L}$. & Dangla & $\mathrm{T}$ & $\mathrm{LC}$ & $\mathrm{N}$ \\
\hline & Vitex parviflora Juss. & Sagat & $\mathrm{T}$ & $\mathrm{LC}$ & $\mathrm{N}$ \\
\hline \multirow[t]{2}{*}{ Lauraceae } & Cinnamomum anacardium Kosterm & Ikmo & $\mathrm{T}$ & $\mathrm{NE}$ & $\mathrm{E}$ \\
\hline & Litsea glutinosa (Lour.) C.B.Rob. & Sablot & $\mathrm{T}$ & $\mathrm{LC}$ & $\mathrm{N}$ \\
\hline \multirow[t]{2}{*}{ Loranthaceae } & Amyema incarnatiflora (Elmer) Danser & Kanonong & $\mathrm{S}$ & $\mathrm{NE}$ & $\mathrm{E}$ \\
\hline & Scurrula parisitica $\mathrm{L}$. & Kankanonong & $\mathrm{S}$ & $\mathrm{NE}$ & $\mathrm{N}$ \\
\hline \multirow{2}{*}{ Lycopodiaceae } & Phlegmariurus phlegmaria (L.) Holub & Aroy-uy & $\mathrm{E}$ & $\mathrm{E}^{*}$ & $\mathrm{~N}$ \\
\hline & Lycopodium sintenisii (Herter) Maxon ex C. Chr. & & $\mathrm{E}$ & $\mathrm{NE}$ & $\mathrm{N}$ \\
\hline \multirow[t]{8}{*}{ Malvaceae } & Grewia bilamellata Gagnep & & $\mathrm{S}$ & $\mathrm{NE}$ & $\mathrm{N}$ \\
\hline & Helicteres hirsuta Lour. & & $\mathrm{S}$ & $\mathrm{NE}$ & $\mathrm{N}$ \\
\hline & Kleinhovia hospital L. & & $\mathrm{T}$ & NE & $\mathrm{N}$ \\
\hline & Pterocymbium tinctorium (Blanco) Merr. & & $\mathrm{T}$ & $\mathrm{LC}$ & $\mathrm{N}$ \\
\hline & Pterospermum diversifolium Blume & Bayok & $\mathrm{T}$ & $\mathrm{LC}$ & $\mathrm{N}$ \\
\hline & Pterospermum obliquum Blanco & Bayok-bayok & $\mathrm{T}$ & $\mathrm{LC}$ & $\mathrm{E}$ \\
\hline & Sterculia foetida $\mathrm{L}$. & Bangar & $\mathrm{T}$ & NE & $\mathrm{N}$ \\
\hline & Urena lobata $\mathrm{L}$. & Maratinta & $\mathrm{S}$ & $\mathrm{NE}$ & $\mathrm{N}$ \\
\hline Melastomataceae & Memecylon edule Roxb. & Candon & $\mathrm{T}$ & NE & $\mathrm{N}$ \\
\hline \multirow[t]{2}{*}{ Meliaceae } & Dysoxylum gaudichaudianum (A.Juss.) Miq. & Igyo & $\mathrm{T}$ & $\mathrm{LC}$ & $\mathrm{N}$ \\
\hline & Swietenia mahagoni (L.) Jacq. & Mahogany & $\mathrm{T}$ & NT & $\mathrm{N}$ \\
\hline \multirow[t]{6}{*}{ Moraceae } & Artocarpus blancoi (Elmer) Merr. & Antipolo & $\mathrm{T}$ & VU & $\mathrm{E}$ \\
\hline & Artocarpus ovatus Blanco & & $\mathrm{T}$ & $\mathrm{NE}$ & $\mathrm{E}$ \\
\hline & Ficus nota (Blanco) Merr. & Tibbeg & $\mathrm{T}$ & $\mathrm{LC}$ & $\mathrm{N}$ \\
\hline & Ficus parvifolia (Miq.) Miq. & Balliti & $\mathrm{T}$ & $\mathrm{NE}$ & $\mathrm{N}$ \\
\hline & Ficus septica Burm.f. & Raya-raya & $\mathrm{T}$ & $\mathrm{LC}$ & $\mathrm{N}$ \\
\hline & Ficus variegata Blume & $\begin{array}{l}\text { Tagisang- } \\
\text { bayawak }\end{array}$ & $\mathrm{T}$ & $\mathrm{LC}$ & $\mathrm{N}$ \\
\hline \multirow[t]{7}{*}{ Myrtaceae } & Eucalyptus camaldulensis Dehnh. & Kamal & $\mathrm{T}$ & NT & $\mathrm{N}$ \\
\hline & Eucalyptus deglupta Blume & Bagras & $\mathrm{T}$ & VU & $\mathrm{N}$ \\
\hline & Leptospermum amboinense Blume & Marabayawas & $\mathrm{T}$ & $\mathrm{NE}$ & $\mathrm{N}$ \\
\hline & Syzygium cumini (L.) Skeels & Lumboy & $\mathrm{T}$ & $\mathrm{LC}$ & $\mathrm{N}$ \\
\hline & Syzygium ilocanum (Merr.) Merr. & Panlungbuyen & $\mathrm{T}$ & NE & $\mathrm{E}$ \\
\hline & Syzygium simile (Merr.) Merr. & Panlungbuyen & $\mathrm{T}$ & $\mathrm{NE}$ & $\mathrm{N}$ \\
\hline & Syzygium xanthophyllum (C.B.Rob.) Merr. & Panlungbuyen & $\mathrm{T}$ & $\mathrm{NE}$ & $\mathrm{E}$ \\
\hline Nepenthaceae & Nepenthes alata Blanco & Batbatidor & $\mathrm{E}$ & $\mathrm{LC}$ & $\mathrm{E}$ \\
\hline \multirow[t]{2}{*}{ Oleaceae } & Chionanthus coriaceus (S.Vidal) Yuen P.Yang \& S.Y.Lu & Bangbanglo & $\mathrm{S}$ & $\mathrm{NE}$ & $\mathrm{N}$ \\
\hline & Fraxinus griffithii C.B.Clarke & & $\mathrm{T}$ & $\mathrm{LC}$ & $\mathrm{E}$ \\
\hline Opiliaceae & Champereia manillana (Blume) Merr. & Pannalayapen & $\mathrm{S}$ & $\mathrm{LC}$ & $\mathrm{N}$ \\
\hline \multirow[t]{9}{*}{ Orchidaceae } & Corybas ramosianus J.Dransf. & & $\mathrm{H}$ & $\mathrm{E}^{*}$ & $\mathrm{E}$ \\
\hline & Dendrobium uniflorum Griff & Sangumay & $\mathrm{E}$ & $\mathrm{NE}$ & $\mathrm{N}$ \\
\hline & Pteroceras philippinense (Ames) Garay & & $\mathrm{E}$ & $\mathrm{NE}$ & $\mathrm{E}$ \\
\hline & Plocoglottis bicomata L.O.Williams & & $\mathrm{E}$ & NE & $\mathrm{E}$ \\
\hline & Spathoglottis kimballiana Hook.f. & $\begin{array}{l}\text { Yellow round } \\
\text { orchids }\end{array}$ & $\mathrm{H}$ & NE & $\mathrm{N}$ \\
\hline & Spathoglottis vanoverberghii Ames & & $\mathrm{H}$ & $\mathrm{NE}$ & $\mathrm{E}$ \\
\hline & Trichoglottis amesiana L.O.Williams & & $\mathrm{E}$ & $\mathrm{NE}$ & $\mathrm{E}$ \\
\hline & Thrixpermum elongatum Ames & & $\mathrm{E}$ & NE & $\mathrm{E}$ \\
\hline & Thrixspermum nicolasiorum Calaramo, Cootes \& Naive & Nicolas Orchid & $\mathrm{E}$ & $\mathrm{NE}$ & $\mathrm{E}$ \\
\hline Orobanchaceae & Lindenbergia philippensis (Cham. \& Schltdl.) Benth. & & $\mathrm{S}$ & $\mathrm{NE}$ & $\mathrm{N}$ \\
\hline Pandanaceae & Pandanus tectorius Soland & Pandan siitan & $\mathrm{T}$ & $\mathrm{LC}$ & $\mathrm{N}$ \\
\hline Pentaphyllaceae & Ternstroemia toquian (Blanco) Fern. & & $\mathrm{T}$ & $\mathrm{LC}$ & $\mathrm{N}$ \\
\hline \multirow[t]{7}{*}{ Phyllanthaceae } & Antidesma ghaesembilla Gaertn. & Arusip & $\mathrm{T}$ & $\mathrm{LC}$ & $\mathrm{N}$ \\
\hline & Antidesma montanum Blume & Bignay pugo & $\mathrm{T}$ & $\mathrm{LC}$ & $\mathrm{N}$ \\
\hline & Flueggea virosa (Roxb. ex Willd.) Royle & & $\mathrm{T}$ & $\mathrm{LC}$ & $\mathrm{N}$ \\
\hline & Glochidion psidioides C.B.Rob & & $S$ & NE & $\mathrm{E}$ \\
\hline & Phyllanthus cf. blancoanus Mull.Arg. & & $\mathrm{S}$ & $\mathrm{NE}$ & $\mathrm{E}$ \\
\hline & Phyllanthus erythrothrichus C.B.Rob. & & $\mathrm{S}$ & $\mathrm{NE}$ & $\mathrm{E}$ \\
\hline & Phyllanthus reticulatus Poir & & $\mathrm{S}$ & NE & $\mathrm{N}$ \\
\hline Pittosporaceae & Pittosporum moluccanum (Lam.) Miq. & & $\mathrm{T}$ & $\mathrm{NE}$ & $\mathrm{N}$ \\
\hline Plantaginaceae & Bacopa monnieri (L.) Wettst & & $\mathrm{H}$ & LC & $\mathrm{N}$ \\
\hline
\end{tabular}




\begin{tabular}{|c|c|c|c|c|c|}
\hline \multirow[t]{6}{*}{ Poaceae } & Dinochloa acutiflora (Munro) S.Dransf. & Bikal & $\mathrm{T}$ & OTS* & $\mathrm{N}$ \\
\hline & Gigantochloa levis (Blanco) Merr. & Bolo & $\mathrm{T}$ & NE & $\mathrm{N}$ \\
\hline & Imperata cylindrica (L.) P.Beauv. & Kogon & $\mathrm{H}$ & $\mathrm{LC}$ & $\mathrm{N}$ \\
\hline & Sacharrum officinarum $\mathrm{L}$. & Ledda & $\mathrm{T}$ & NE & $\mathrm{N}$ \\
\hline & Schizostachyum lumampao (Blanco) Merr. & Bolo & $\mathrm{T}$ & $\mathrm{NE}$ & $\mathrm{E}$ \\
\hline & Themeda intermedia (Hack.) Bor & & $\mathrm{H}$ & $\mathrm{NE}$ & $\mathrm{N}$ \\
\hline \multirow[t]{2}{*}{ Podocarpaceae } & Podocarpus costalis C.Presl & Igem dagat & $\mathrm{T}$ & $\mathrm{E}^{*} / \mathrm{E}$ & $\mathrm{N}$ \\
\hline & Podocarpus polystachyus R.Br. ex Endl. & Igem & $\mathrm{T}$ & VU*/VU & $\mathrm{N}$ \\
\hline \multirow[t]{3}{*}{ Primulaceae } & Ardisia elliptica Thunb. & Lumamani & $\mathrm{T}$ & $\mathrm{NE}$ & $\mathrm{N}$ \\
\hline & Ardisia pyramidalis (Cav.) Pers. & & $\mathrm{S}$ & NE & $\mathrm{N}$ \\
\hline & Myrsine oblongibacca (Merr.) Pipoly & & $\mathrm{S}$ & $\mathrm{NE}$ & $\mathrm{E}$ \\
\hline \multirow[t]{3}{*}{ Pteridaceae } & Adiantum philippense $\mathrm{L}$. & & $\mathrm{E}$ & NE & $\mathrm{N}$ \\
\hline & Ceratopteris thalictroides (L.) Brongn. & Water Sprite & $\mathrm{E}$ & $\mathrm{LC}$ & $\mathrm{N}$ \\
\hline & Pteris vittata $\mathrm{L}$. & & $\mathrm{E}$ & LC & $\mathrm{N}$ \\
\hline Rhamnaceae & Ziziphus talanai (Blanco) Merr. & Talanay & $\mathrm{T}$ & OTS*/VU & $\mathrm{E}$ \\
\hline \multirow{18}{*}{ Rubiaceae } & Argostemma solaniflorum Elmer & & $\mathrm{H}$ & NE & $\mathrm{N}$ \\
\hline & Antirhea microphylla (Bartl. ex DC.) Merr. & & $\mathrm{S}$ & $\mathrm{NE}$ & $\mathrm{E}$ \\
\hline & Canthium sp. & & $\mathrm{S}$ & NE & $\mathrm{N}$ \\
\hline & Ixora cummingiana S. Vidal & Cuming Santan & $\mathrm{S}$ & $\mathrm{NE}$ & $\mathrm{E}$ \\
\hline & Ixora philippinensis Merr. & Mutang-ulang & $\mathrm{S}$ & NE & $\mathrm{N}$ \\
\hline & Kanapia monstrosa (A.Rich) Arriola \& Alejandro & Santan siitan & $\mathrm{T}$ & $\mathrm{NE}$ & $\mathrm{E}$ \\
\hline & Morinda citrifolia $\mathrm{L}$. & Apatot & $\mathrm{T}$ & $\mathrm{NE}$ & $\mathrm{N}$ \\
\hline & Mussaenda philippica A. Rich & Musaenda & $\mathrm{T}$ & $\mathrm{LC}$ & $\mathrm{E}$ \\
\hline & Nauclea orientalis (L.) L. & Bulala & $\mathrm{T}$ & $\mathrm{LC}$ & $\mathrm{N}$ \\
\hline & Neonauclea bartlingii var. cumingiana (S.Vidal) Ridsdale & Bulala Bantay & $\mathrm{T}$ & $\mathrm{LC}$ & $\mathrm{E}$ \\
\hline & Oldenlandia corymbosa $\mathrm{L}$. & & $\mathrm{H}$ & LC & $\mathrm{N}$ \\
\hline & Paederna foetida $\mathrm{L}$. & & $\mathrm{V}$ & $\mathrm{NE}$ & $\mathrm{N}$ \\
\hline & Pyrostria triflora Arriola, Calaramo \& Alejandro & $\begin{array}{l}\text { Psychotria } \\
\text { Sigatlo }\end{array}$ & $\mathrm{S}$ & NE & $\mathrm{E}$ \\
\hline & Psychotria luzoniensis (Cham. \& Schltdl.) Fern.-Vill & Luzon Psychotria & $\mathrm{S}$ & NE & $\mathrm{E}$ \\
\hline & $\begin{array}{l}\text { Spermacoce exilis (L.O Williams) C.D. Adams ex W.C. } \\
\text { Burger \& C.M. Taylor }\end{array}$ & & $\mathrm{H}$ & $\mathrm{NE}$ & $\mathrm{N}$ \\
\hline & Tarrenoidea wallichii (Hook. F.) Tirveng. \& Sastre & & $\mathrm{T}$ & $\mathrm{NE}$ & $\mathrm{N}$ \\
\hline & Timonius ternifolius (Bartl. ex DC.) Fern. & & $\mathrm{T}$ & $\mathrm{NE}$ & $\mathrm{E}$ \\
\hline & Wendlandia luzoniensis D.C. y & Maratabako & $\mathrm{T}$ & $\mathrm{NE}$ & $\mathrm{N}$ \\
\hline Rutaceae & Lunasia amara Blanco & & $\mathrm{S}$ & $\mathrm{LC}$ & $\mathrm{N}$ \\
\hline \multirow[t]{2}{*}{ Salicaceae } & Casearia grewiifolia Vent. & & $\mathrm{T}$ & $\mathrm{LC}$ & $\mathrm{N}$ \\
\hline & Scolopia luzonensis (C.Presl) Warb. & & $\mathrm{T}$ & $\mathrm{NE}$ & $\mathrm{N}$ \\
\hline \multirow[t]{3}{*}{ Sapindaceae } & Dodonaea viscosa (L.) Jacq. & & $\mathrm{S}$ & $\mathrm{NE}$ & $\mathrm{N}$ \\
\hline & Guioa koelreuteria (Blanco) Merr. & Owas & $\mathrm{T}$ & $\mathrm{LC}$ & $\mathrm{N}$ \\
\hline & Sapindus saponaria $\mathrm{L}$. & Kusibeng & $\mathrm{T}$ & LC & $\mathrm{N}$ \\
\hline \multirow[t]{3}{*}{ Sapotaceae } & Palaquium philippense (Perr.) C.B.Rob. & Paak-palak & $\mathrm{T}$ & VU*/VU & $\mathrm{E}$ \\
\hline & Planchonella duclitan (Blanco) Bakh.f. & Duklitan & $\mathrm{T}$ & LC & $\mathrm{N}$ \\
\hline & Xantolis parvifolia (A.DC.) Royen & Siitan & $\mathrm{T}$ & $\mathrm{NE}$ & $\mathrm{E}$ \\
\hline \multirow[t]{2}{*}{ Schizaeaceae } & Lygodium circinatum (Burm. f.) Sw. & Nito & $\mathrm{E}$ & NE & $\mathrm{N}$ \\
\hline & Lygodium japonicum (Thunb.) Sw. & Nito puraw & $\mathrm{E}$ & NE & $\mathrm{N}$ \\
\hline Thymelaeaceae & Wikstroemia indica (L.) C.A.Mey. & Salago & $\mathrm{S}$ & $\mathrm{NE}$ & $\mathrm{N}$ \\
\hline Urticaceae & Oreocnide trinervis (Wedd.) Miq. & Budobudo & $\mathrm{T}$ & NE & $\mathrm{N}$ \\
\hline Verbenaceae & Lantana camara $\mathrm{L}$. & Bangbangsit & $\mathrm{S}$ & NE & $\mathrm{N}$ \\
\hline Zingiberaceae & Alpinia brevilabris C.Presl & Maralaya & $\mathrm{H}$ & NE & $\mathrm{E}$ \\
\hline
\end{tabular}




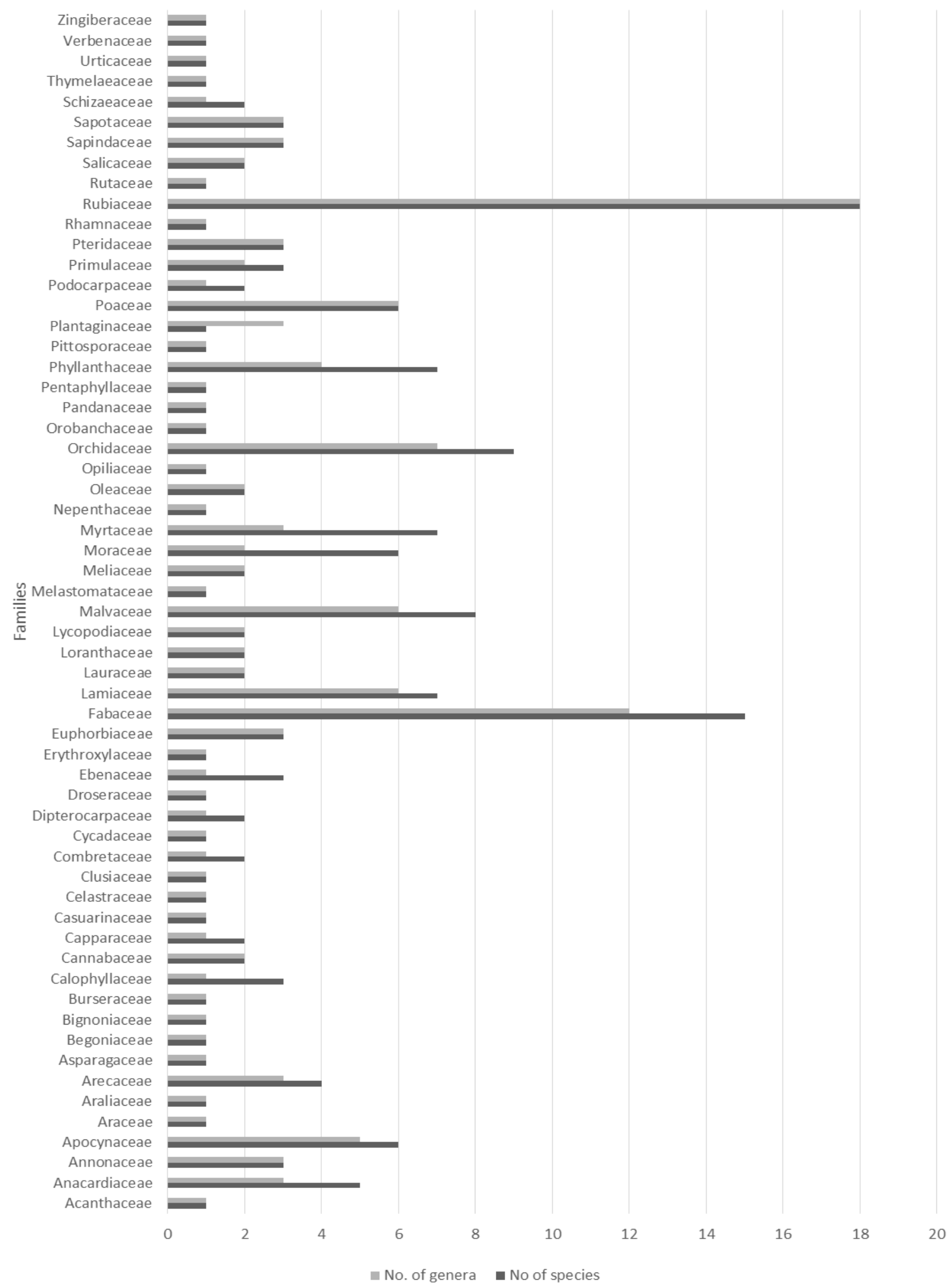

Figure 3. Representation of vascular families and species recorded in MINFWR, Northwestern Luzon, Philippines 


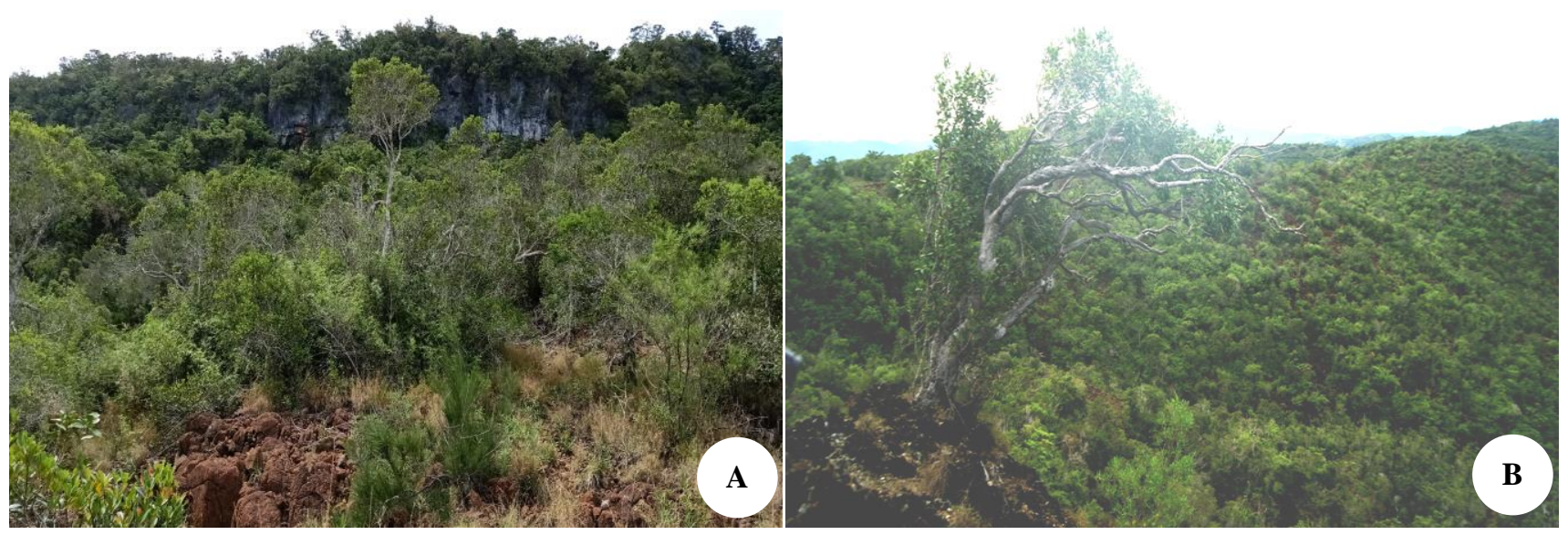

Figure 4. The panoramic view of MINFWR, Northwestern Luzon, Philippines showing the dominant Podocarpus species in the rocky ecosystem. (A) During the dry season, (B) Wet season

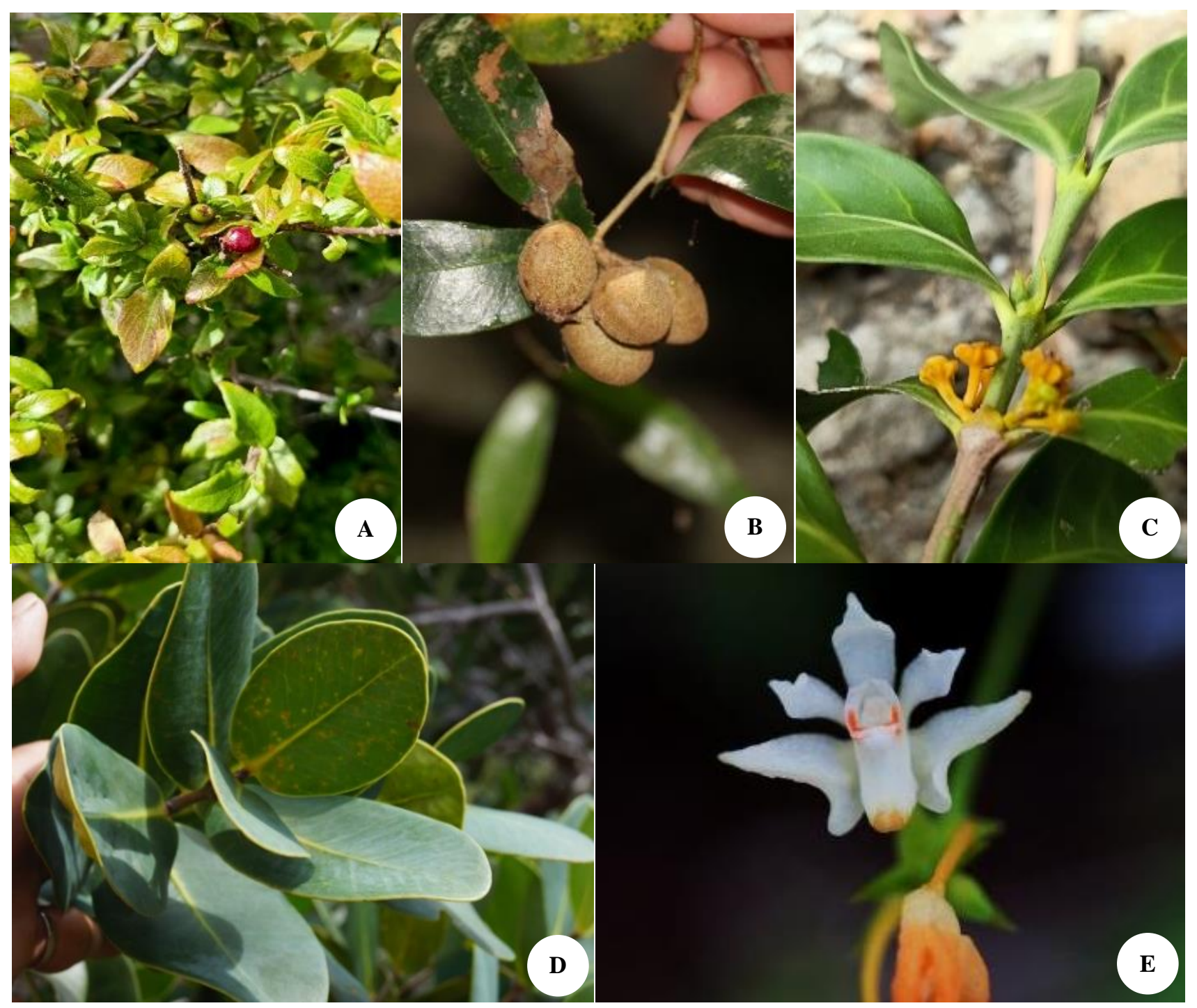

Figure 5. The narrow endemic species recorded in MINFWR, Northwestern Luzon, Philippines. A. Antirhea microphylla showing the leaves and fruits, B. Cyanometra warburgii fruiting branch, C. Pyrostria triflora reproductive branch showing the developing flower, D. Syzygium ilocanum vegetative part, E. Thrixspermum nicolasiorum flower. Photographs by M.A.Batuyong and M.A. Calaramo. 


\section{Endemism}

Assessing the geographical distribution of plant species is essential in defining the biodiversity value of an area which typically measured in terms of species richness and the number of endemic and threatened species present (Clergue et al. 2005; Bruchmann 2014). Species confined or restricted to a particular site should be provided with specific conservation management strategies as they are more vulnerable to disturbance and higher risk for loss due to their narrow range (Haq et al. 2010). Moreover, for their high value and importance in genetic diversity.

Of the 171 identified plants to species level, 49 species or $28.65 \%$ are Philippine endemics (Table 3; Figure 3). This is $1.03 \%$ out of the 4742 Philippine endemics identified based on Co's Digital Flora of the Philippines data (Pelser et al. 2011 onwards). Remarkably, 5 of which are known to be narrow endemics or have natural habitat confined only in the Province. These include Cyanometra warburgii Harms, Pyrostria triflora Arriola, Calaramo \& Alejandro, Syzygium ilocanum (Merr.) Merr., and Thrixspermum nicolasiorum Calaramo, Cootes \& Naïve (Figure 5). Also, to add Antirhea microphylla (Bartl. ex DC. ) Merr., which is supposedly endemic to Ilocos Norte but has extended its distribution in Ilocos Sur.

The Rubiaceae family had the highest number of endemic species (8), followed by Orchidaceae (7), Apocynaceae (4), and Arecaceae (3), Phyllanthaceae (3). Endemism was principally at the species level. The families and most genera in the study were all widespread or broadly found in the tropics. Thus, no family was endemic to the Philippines. The only endemic genus found was the Kanapia of the family Rubiaceae (Arriola et al. 2015). The presence of endemic species in this area also connoted ecological significance and considered a good indication of the status of habitats and vegetation of this limestone forest. It should also be emphasized that categorizing species as endemic is very much dependent on the availability of published biodiversity data, recent taxonomic revisions, nomenclatural changes, and new pieces of evidence from various disciplines used in systematics among others. Thus, estimates of endemism should be interpreted within the context of the methodologies and limitations imposed by contributing factors aforementioned.

Introduced species are also observed in the area, such as the cogon grass Imperata cylindrica (L.) P. Beauv., large leaf Lantana camara L., and Leucaena leucocephala (Lam.) de Wit as listed among Philippine invasive alien species (IAS) (Joshi 2006) and are also included in the Global Compendium of Weeds. These IAS are introduced both by intentional or accidental by human activity from outside its normal territorial range of distribution. Also, control has become difficult because they are prolific, good dispersers (UNEP 2013; DENR-BMB 2016), and can aggressively displace or outnumber indigenous species in their natural habitats, which may result in alteration of ecosystem structure and function (Pysek et al. 2017; Garces
2019). They have been considered as the second most prominent threat to biodiversity after habitat destruction (Bellard et al. 2018). The presence of these species in the PA are noted and can be utilized and integrated for the management strategies as an early recognition effort in the existing monitoring and surveillance programs being implemented.

\section{Assessment of conservation status}

The conservation status of each species was noted and recorded based on the most recent recommendations issued by DENR as Administrative Order No. 2017-01 and the listing of threatened species of the IUCN as reference. This was carried out to provide a foundation on their conservation status, thereby, necessary in the establishment of their protection and conservation as priority measures locally and globally.

Out of the 173 total species identified in the limestone forest, DENR-DAO 17 and IUCN Red list considered 14 and 13 threatened plant species, respectively. Following the list of DAO 2017-11 marked $1 \mathrm{CR}, 3 \mathrm{EN}, 7 \mathrm{VU}$, and 3 OTS while IUCN Redlist included 4 EN, 7 VU, 2 NT, and 55 LC. Both DENR and IUCN categorized Areca ipot Becc., P. polystachyus, and Palaquium philippense (Perr.) C.B. Rob. as VU species, while $P$. costalis as EN. For the other species, Corybas ramosianus J.Dransf. and Phlegmariurus phlegmaria (L.) Holub were reported by DENR as EN, whereas Shorea astylosa Foxw. as CR, although these Dipterocarp species are frequent, as reported by Quimio (2016) in the Samar Island Natural Park. Besides, Cycas riuminiana Porte ex Regel, Dracontomelon dao (Blanco) Merr \& Rolfe, Diospyrus pilosanthera Blanco, Radermachera coriacea Merr. and Shorea contorta $\mathrm{S}$. Vidal were included by DENR all under VU. In contrast with IUCN, S. astylosa is considered as EN. Moreover, IUCN assessment for $C$. riuminiana and $S$. contorta are EN and LC, respectively. Lastly, IUCN evaluated the following species, Pterocarpus indicus Willd. as EN while Artocarpus blancoi Merr., Eucalyptus deglupta Blume, and Terminalia nitens C. Presl. were considered as VU, but these species were not included in DENR DAO-17. Lastly, both Eucalyptus camaldulensis Dehnh. and Swietenia mahagoni (L.) Jacq. were categorized as NT. The rest of the plant species were marked as not evaluated (NE) (Table $3)$. As ascertained during this study, IUCN and DENR have different categories for a selected species, and this can be because of their variations in scope or level of assessment (Villanueva and Buot 2015).

Moreover, it is interesting to note the following species, i.e., A. ipot, A. blancoi, C. ramosianus, C. riuminiana, $P$. philippense, $R$. coriacea, S. astylosa, and Z. talanai are all endemic plants that have been identified as threatened by DENR and IUCN (v. 3.1), therefore, an immediate and ensured appropriate management and monitoring strategies should as well be developed to warrant for their protection and the continued survival of their population. 
Table 3. Summary of Threatened species recorded in MINWFR, Northwestern Luzon, Philippines

\begin{tabular}{llll}
\hline Categories & IUCN & DENR & Total \\
\hline Critically Endangered (CR) & 0 & 1 & 1 \\
Endangered (EN) & 4 & 3 & $* 5$ \\
Vulnerable (VU) & 7 & 7 & $* 9$ \\
Other Threatened (OT) & 0 & 3 & $* 3$ \\
Near Threatened (NT) & 2 & 0 & 2 \\
Least Concern (LC) & 55 & 0 & 55 \\
Data Deficient (DD) & 0 & 0 & \\
Not Evaluated (NE) & 103 & 157 & 18 \\
Total (CR, EN, VU, OT) & \multicolumn{2}{l}{} \\
$\begin{array}{l}\text { Note: } \text { * If IUCN or DENR listed same plant species, the plant is } \\
\text { counted as one }\end{array}$
\end{tabular}

During the fieldworks, several threats were also observed, such as the expansion of some areas into agricultural lands, built infrastructures like transmission lines at different locations within the PA, extensive dam construction, and conversion of the pristine ecosystem to residential areas. Added to this is the concrete road that intersects the middle portion of the protected area, which serves as access to the Paredes Air Station and Brgys. Sulbec, Salpad, and Sapat. The unregulated tourist visits, resulting in improper waste disposal and picnic litters within the area. And other human activities such as timber poaching, bonsai collection, wildlife hunting, and mortar rock collection.

The protected area has much potential for ecotourism because of its caves, springs, waterfalls, and view overlooking the Municipality of Pasuquin and various livelihood programs and projects, with its rich biodiversity. However, this critical area will be lost without proper management and monitoring.

In conclusion, the result of the preliminary checklist of vascular plants in MINFWR revealed that it is home to 173 plant species, belonging to 140 genera distributed in 59 families. Forty-nine or $28.65 \%$ of the identified species are exclusively found in the country, which represents $1.03 \%$ from species identified as Philippine endemics. Based on IUCN criteria and DENR records, 18 species are threatened, one species is recorded as critically endangered, five as endangered, nine vulnerable, three other threatened, two near threatened, and fifty-five as least concern and the rest are not evaluated. Other noteworthy species present in the area, which are considered narrow endemics, include $A$. microphylla, C. warburgii, S. ilocanum, T. nicolasiorum, and $P$. triflora. The presence of an unusual spinescent Rubiaceae species initially placed under genus Canthium is being examined for its identity.

Though there were only a few documented plant species under threat, it cannot be denied, it is justifiable to consider MINFWR as an ecologically critical area. Also, because of the presence of invasive alien species and naturalized species in the area. There is a need to intensify further research to strengthen the conservation plans and programs and management intervention being implemented. All stakeholders should work together to safeguard and ensure the balance between sustainably protecting the biodiversity of this area, with economic development. All sectors must take proactive actions and participate, coupled with strong support to monitor, ensure, and evaluate the progress and implementation of programs.

With the concerted efforts and extensive works done, the research group finally attained the initial target of this study, and further steps are being considered and will be undertaken, such as the reassessment and continued monitoring of this area in the years to come.

Several management options can be worked out in the area, such as the development and dissemination of Instruction, Education, and Communication (IEC) materials of the species and the placing of labels and other relevant information regarding the species, particularly those along the commonly traversed trail as part of the ecotourism and general public awareness campaign of PAMB to gain broader support for its protection and eventual awareness and appreciation.

Another is the creation of the distribution map of threatened species. This map bears the critical spots where these threatened species are located and the application of a geographic information system (GIS) as a tool to map the specific locations of threatened species so that easy monitoring can be carried out immediately. The inventory of other plants, such as bryophytes, is also recommended for future studies. Also, it should be considered to do the detailed taxonomic work of the flora of MINFWR to provide identification keys to all species and, in general, to contribute to the update of Philippine diversity. This study has significantly increased the plant biodiversity data of the protected area that can be used to intensify environmental regulations and strengthen management programs on the utilization and conservation of MINFWR.

\section{ACKNOWLEDGEMENTS}

The authors would like to thank the directors and curators of CAHUP, HNUL, PNH, and USTH for granting access to the herbarium collections, DENR Regional Office 1, PENRO-Ilocos Norte, CENR Office-Bangui and the MINWFR Protected Area Management Board for the opportunity to document and survey the area. The first author thanks the Commission on Higher Education (CHED) and Mariano Marcos State University (MMSU) for the Scholarship Grant.

\section{REFERENCES}

Amoroso VB, Aspiras RA. 2011. Hamiguitan Range: A sanctuary for native flora. Saudi J Biol Sci 18 (1): 7-16. DOI: 10.1016/j.sjbs.2010.07.003

Arriola AH, Paraguison LD, Alejandro GJD. 2015. Kanapia (Vanguerieae): a new endemic genus of Philippine Rubiaceae. Plant Syst Evol 302 (7): 911-920. DOI: 10.1007/s00606-016-1317-5

Bacudo AA, Mores MCL, Palaganas JA, Barrameda PG, Sanchez KP, Salcedo PG. 2006. Biodiversity assessment and GIS mapping of MinSCAT forest reservation. MinSCAT Report 34: 2007-01.

Bellard C, Jeschke JM, Leroy B, Mace GM. 2018. Insights from modeling studies on how climate change affects invasive alien species geography. Ecol Evol 8 (11): 5688-5700. 
Brinkmann R, Parise M. 2012. Karst environments: problems, management, human impacts, and sustainability. An introduction to the special issue. J Cave Karst Stud 74 (2): 135-136.

Brown RM, Siler CD, Oliveros CH, Esselstyn JA, Diesmos AC, Hosner PA, Linkem CW, Barley AJ, Oaks, JM, Sanguila MB, Welton LJ, Blackburn DC, Moyle RG, Peterson T, Alcala AC. 2013. Evolutionary processes of diversification in a model island archipelago. Ann Rev Ecol Evol Syst 44: 411-435

Bruchmann I. 2014. Facing the Biodiversity Challenge: Plant Endemism as an Appropriate Biodiversity Indicator. In: Concepts and Values in Biodiversity. Routledge Studies in Biodiversity Politics and Management, Routledge, UK.

Calaramo MA, Cootes J, Palting V. 2016. Cleisostoma iloconense. A new species from Northwestern Luzon, Philippines. Die Orchidee 2 (8): 1 4.

Clements R, Sodhi NS, Schilthuizen, M, Ng PKL. 2006. Limestone karsts of Southeast Asia: Imperiled arks of biodiversity. Bioscience 56 (9): 733-742. DOI: 10.1641/0006-3568 (2006)56[733:lkosai]2.0.co;2.

Clergue B, Amiaud B, Pervachon F, LasserreJoulin F, Plantureux S. 2005. Biodiversity: function and assessment in agricultural areas: A review. Agron Sustain Dev 25: 1-15.

Conservation International (CI). 2020. CI website. www.conservation.org/global/philippines

Convention on Biological Diversity (CBD). 2020. Philippines Country Profile: Actions taken to achieve the 2020 Aichi Biodiversity Targets. www.cbd.int/countries/profile/default.shtml?country=ph

Dapar MLG, Alejandro GJD, Meve U, Liede-Schumann S. 2020 Ethnomedicinal importance and conservation status of medicinal trees among indigenous communities in Esperanza, Agusan del Sur, Philippines. J Complement Med Res 11 (1): 59-71. DOI: 10.5455/jcmr.2020.11.01.08

Department of Environment and Natural Resources Administrative Order (DAO2017). 2017. Updated national list of threatened Philippine plants and their categories. DAO2017-11 https://server2.denr.gov.ph/uploads/ rmdd/dao-2017-11.pdf.

Department of Environment and Natural Resources- Biodiversity Management Bureau (DENR-BMB). 2015. Guidebook to Protected Areas of the Philippines. Biodiversity Management Bureau, Department of Environment and Natural Resources, Philippines.

Department of Environment and Natural Resources- Biodiversity Management Bureau (DENR-BMB). 2016. Course Manual on Invasion Biology. Department of Environment Biodiversity Management Bureau and Natural Resources, Quezon City, Philippines.

Department of Environment and Natural Resources- Biodiversity Management Bureau (DENR-BMB). 2019. Protection and Conservation Strategy and Action Plan. www.bmb.gov.ph/bmb/CAWED/Proposed_Policy/CMPCSAP_20192028.pdf?cv=1

Department of Environment and Natural Resources, Provincial Environment and Natural Resources Office- Ilocos Norte. (DENRPENRO). 2018. Metropolitan Ilocos Norte Watershed Forest Reserve Management Plan. Department of Environment and Natural Resource Provincial - Environment and Natural Resources Office, Philippines.

Fernando ES, Suh MH, Lee J, Lee DK. 2008. Forest Formations of the Philippines, ASEAN-Korea Environmental Cooperation Unit (AKECU). GeoBook, South Korea.

Garces JC. 2019. Native and alien plant species inventory and diversity in disturbed forests and its economic value. Global J Environ Sci Manag 5 (2): 249-264

GBIF.org, 1999-onwards. Global Biodiversity Information Facility; GBIF Home Page. https://www.gbif.org.

Govaerts R, Ruhsam M, Anderson L, Robbrecht E, Bridson D, Davis A, Schanzer I, Sonke B. 2006. World checklist of Rubiaceae. Royal Botanic Gardens, Kew. http//: www. Kew.org/wcsp/rubiaceae/.

Haq FU, Ahmad H, Alam M, Ahmad I, Rahatullah. 2010. Species diversity of vascular plants of Nandiar Valley Western Himalaya, Pakistan. Pak J Bot 42: 213-229

IUCN. 2018. The International Union for Conservation of Nature Red List of Threatened Species. Version 2018-1. http://www.iucnredlist.org.

Joshi RC. 2006. Invasive alien species (IAS): Concerns and status in the Philippines. Philippine Rice Research Institute (PhilRice) Maligaya, Science City of Muñoz, Nueva Ecija 3119, Philippines.

JSTOR (Journal Storage). 2000-onwards. Global Plants. https://plants jstor.org/.
Kiew R. 2014. Checklist of vascular plants from Batu Caves, Selangor, Malaysia. Check List (6): 1420-1429. DOI: 10.15560/10.6.1420

Merrill ED. 1925. An Enumeration of Philippine Flowering Plants. Bureau of Printing, Manila.

Mittermeier RA, Turner WR, Larsen FW, Brooks TM, Gascon C. 2011. Global Biodiversity Conservation: The Critical Role of Hotspots in: Biodiversity Hotspots. In: Zachos F, Habel J (eds) Biodiversity Hotspots. Springer, Berlin.

Myers N, Mittermeier RA, Mittermeier CG, Da Fonseca GA, Kent J. 2000. Biodiversity hotspots for conservation priorities. Nature 403 (6772):853-858

Ong PS, Afuang LE, Rosell, Ambal RG. 2002. Philippine Biodiversity Conservation Priorities: A Second Iteration of the National Biodiversity Strategy and Action Plan. Philippine Department of the Environment and Natural Resources, Quezon City.

Ordas JA, Pinarok N, Romeroso R, Alejandro GJD, Banag C. 2019. A checklist or Rubiaceae species from Eastern Samar, Visayas, Philippines. Check List 15: 295-312. DOI: 10.15560/15.2.295.

PCARRD. 2017. Manual on vegetational analysis for grassland and forest ecosystems. Philippine Council for Agriculture, Forestry and Natural Resources and Development. Department of Since and Technology, Los Banos, Philippine.

Pedregosa M, Paguntalan L, Jakosalem P, Lillo E, Rico E, Alban JD, Lorica R, Lastica E, Enricoso, F. 2006. An assessment of the native flora and fauna of Sicogon Island, Panay, Philippines: a consolidated report. DOI: 10.13140/RG.2.1.4720.4244.

Pelser PB, Barcelona JF, Barcelona, Nickrent DL (eds). 2011 onwards. Co's Digital Flora of the Philippines. www.philippineplants.org

Perez-Garcia E, Sevilha A, Meave J, Scariot A. 2009. Floristic differentiation in limestone outcrops of Southern Mexico and Central Brazil; A beta diversity approach. B Soc Bot Mex 84: 45-58.

Posa MRC, Diesmos AC, Sodhi NS, Brooks TM. 2008: Hope for threatened tropical biodiversity: Lessons from the Philippines. BioScience 58: 231-240.

Pysek P, Blackburn TM, Garcia-Berthou E, Perglova I, Rabitsch W. 2017. Displacement and local extinction of native and endemic species. In impact of biological invasions on ecosystem services. Springer Series in Invasion Ecology 12: 157-175. DOI: 10.1007/978-3-319-451213_10

Quimio J. 2016. Floral composition and timber stock of forest in the Samar Island Natural Park. Ann Trop Res 38 (2):30-51.

Replan EL, Malaki AB. 2017. Floral Diversity and Habitat Assessment of Canbantug Forest, Argao, Central Visayas, Cebu, Philippines. Intl J Appl Sci Eng Res (8) 10: 775-780.

Rockstrom J, Steffen W, Noone K, Persson A, III Chapin FS, Lambin EF, Lenton TM, Scheffer M, Folke C, Schellnhuber HJ, Nykvist B, de Wit CA, Hughes T, van der Leew S, Rodhe H, Sorlin S, Synder PK, Costanza R, Svedin U, Falkenmark M, Karlberg L, Corell RW, Fabry VJ, Hansen J, Walker B, Liverman D, Richardson K, Crutzen P, Foley JA. 2009. A Safe operating space for humanity. Nature 461: 472-475.

Saw LG, Chua L, Suhaid M, Yong W, Hamidah M. 2010. Conservation of some rare and endangered plants from Penisular Malaysia. Kew Bull 65: 681-689. DOI: 10.10007/s12225-011-9251-6.

Sinsin B, Assede EM, Adomou AC. 2012. Magnoliophyta, Biosphere of Pendjari, Benin. Check List 8 (4): 642-661.

Sodhi NS, Posa MRC, Lee TM, Bickford D, Koh LP, Brook BW. 2010. The state and conservation of Southeast Asian biodiversity. Biodivers Conserv 19: 317-328.

Stockli V, Wipf S, Nilsson C, Tixen C. 2011. Using historical plant surveys to track biodiversity on mountain summits. Plant Ecol Divers 4: 415-425.

The Plant List. 2010-onwards. Verson 1. http://www.theplantlist.org/.

Tolentino PJS, Natividad JRL, Delos Angeles M, Fernandez DAP, Villanueva ELC, Obeña RDR, Buot IE Jr. 2020. Review: Biodiversity of forests over limestone in Southeast Asia with Emphasis on the Philippines. Biodiversitas 21 (4): 1597-1613. DOI: 10.13057/biodiv/d210441

UNEP. 2012. Green economy advisory services. United National Environment Program, The Philippines.

UNEP. 2013. The enabling policy and institutional environment for invasive alien species management in the Philippines. Draft Report Submitted to CABI under the UNEP/GEF Project: Removing Barriers to Invasive Species Management in Production and Protection Forests in Southeast Asia (UNEP/GEF 0515 Project). Protected Areas and 
Wildlife Bureau-Department of Environment and Natural Resources, Quezon City, Philippines.

Villanueva ELC, Buot IE Jr. 2015. Threatened plant species of Mindoro, Philippines. IAMURE Int $\mathrm{J}$ Ecol Con 14: 168-189. DOI: 10.7718/ijec.v14i1.901
Whitmore TC. 1984. Tropical Rain Forests of The Far East. Oxford University Press, Oxford.

Yap SL. 2010. Phylogeography and Demography of Common Plant Species from the Philippines. [Dissertation]. The University of Michigan, Ann Arbor, MI. [USA] 